\title{
LAS COLECCIONES DE AVES Y MAMÍFEROS DEL MUSEO NACIONAL DE CIENCIAS NATURALES (CSIC)
}

\author{
J. Barreiro (*)
}

\begin{abstract}
RESUMEN
Se presenta información sobre el contenido taxonómico y geográfico de las colecciones de aves y mamíferos del Museo Nacional de Ciencias Naturales, así como del sistema de acceso a ellas. También se realiza una breve reseña histórica de la génesis de ambas colecciones.

Palabras clave: Museología; Colecciones de Aves; Colecciones de Mamíferos; Museo Nacional de Ciencias Naturales de Madrid; Conservación de Colecciones.
\end{abstract}

\section{ABSTRACT \\ The Bird and Mammal collections of the Spanish Museo Nacional de Ciencias Naturales}

In this paper the taxonomic and geographic contents of the Spanish Museo Nacional de Ciencias Naturales Bird and Mammal collections, as well as its system and is revised. A brief summary of the history of these collections is also presented.

Key words: Museology; Bird Collections; Mammal Collections; Natural History Collections curation; Spanish Museo Nacional de Ciencias Naturales.

\section{Introducción histórica}

Antes de realizar una breve reseña histórica de la génesis de estas colecciones, se van a considerar de forma muy concisa los tipos de colecciones a tratar. Las Colecciones de Exhibición (montadas o naturalizadas) están compuestas por ejemplares que se presentan al público en posturas naturales y también incluyen los esqueletos montados. Las Colecciones Científicas están formadas por ejemplares muy bien documentados desde el punto de vista taxonómico y geográfico, y sirven de substrato para la investigación biológica.

La historia de estas colecciones corre paralela a la del Museo y se puede dividir, en cuatro periodos: Real Gabinete de Historia Natural (1771-1808); de 1814 a finales del siglo XIX; desde principios del siglo $\mathrm{XX}$ hasta 1936; y desde la última guerra civil (1936) hasta nuestros días. Para un mayor conocimiento de la historia de su formación consultar Barreiro (1992).

Del Real Gabinete, desafortunadamente quedan muy pocas piezas, como por ejemplo, la piel y el esqueleto del Elefante Indio (Elephas maximus), un Pangolín (Manis tricuspis) naturalizado y un cráneo y varios huesos de un Cachalote (Physeter macrocephalus) remitido de América en 1776. De las colecciones originales compradas a Franco Dávila no parece conservarse ningún elemento.

Del segundo periodo proceden algunos de los ejemplares más antiguos (1836 y 1838) y bellos que tenemos en las colecciones de aves, como es el caso de ocho "Fanales" formados por pájaros naturalizados dispuestos sobre una especie de árbol. En las colecciones de mamíferos se obtuvo la colección de

* Museo Nacional de Ciencias Naturales. José Gutiérrez Abascal, 2. 28006 Madrid. Correo electrónico: jbarreiro@mncn.csic.es 
Tabla 1.- Forma de conservación de los ejemplares de las colecciones. La primera cifra representa el total de ejemplares y la cifra entre paréntesis los ejemplares españoles.

\begin{tabular}{lcc}
\hline & AVES & MAMÍFEROS \\
\hline PIELES & $18.808(6.057)$ & $8.346(5.112)$ \\
ESQUELETOS & $5.999(1.712)$ & $8.219(4.286)$ \\
FLUIDOS & $1.911(635)$ & $3.271(1.805)$ \\
HUEVOS Y/O NIDOS & $1.529(1.007)$ & - \\
CUERNAS & - & $40(3)$ \\
OTROS & $30(13)$ & $75(15)$ \\
\hline
\end{tabular}

marsupiales australianos, entre los que destacamos el Lobo marsupial (Thylacinus cynocephalus) especie hoy extinguida. En este periodo se formaron la mayoría de las colecciones de fauna no ibérica que tenemos en la actualidad (Asia, Oceanía y América) y se obtuvo la colección de esqueletos montados.

Durante el tercer periodo se formaron la mayor parte de las colecciones de aves y mamíferos de fauna ibérica, las colecciones naturalizadas actuales y las africanas y europeas.

En el cuarto periodo, las composición de las colecciones es fundamentalmente la que se describe en el siguiente apartado. Cabe destacar la donación que la Comunidad de Madrid hace al Museo de una importante colección de vertebrados de la fauna ibérica (Colección de la Unidad de Zoología Aplicada, UZA), que duplicó el número de ejemplares de la colección de mamíferos y que permite al museo contar con una razonablemente completa y documentada representación de nuestra fauna.

\section{Composición de las colecciones}

LA COLECCIÓN DE AVES. - Consta de aproximadamente 30.000 ejemplares cuya forma de conservación se presenta en la Tabla 1. Esta colección se encuentra inventariada en un $76.2 \%$ y este inventario está totalmente informatizado. El $23.8 \%$ restante son los ejemplares en fase de preparación (6.5\%) y especímenes conservados en alcohol de los que existe un inventario a nivel de familia (17.3\%).

Respecto a la composición taxonómica de la colección, hay representación de los 27 órdenes de la clase Aves (Peters, 1931-1987, Howard \& Moore, 1991), ver Tabla 2. En total están representadas 146 familias (Tabla 3), 1.205 géneros y 2.690 especies, aunque todavía resta por revisar taxonómicamente la colección.

La gran mayoría del material procede del territorio español, y entre los fondos extranjeros se
Tabla 2.- Número de ejemplares de cada Orden representado en las colecciones de aves y de mamíferos.

\begin{tabular}{|c|c|c|c|}
\hline \multicolumn{2}{|l|}{ AVES } & \multicolumn{2}{|c|}{ MAMÍFEROS } \\
\hline STRUTIONIFORMES & 20 & MONOTREMATA & 6 \\
\hline RHEIIFORMES & 22 & MARSUPIALIA & 69 \\
\hline CASUARIIFORMES & 9 & EDENTATA & 43 \\
\hline APTERYGIFORMES & 1 & INSECTIVORA & 1.129 \\
\hline TINAMIFORMES & 36 & SCANDENTIA & 16 \\
\hline SPHENISCIFORMES & 9 & DERMOPTERA & 8 \\
\hline GAVIIFORMES & 10 & CHIROPTERA & 1.610 \\
\hline PODICIPEDIFORMES & 187 & PRIMATES & 296 \\
\hline PROCELLARIIFORMES & 89 & CARNIVORA & 1.181 \\
\hline PELECANIFORMES & 130 & CETACEA & 21 \\
\hline CICONIIFORMES & 489 & SIRENIA & 3 \\
\hline ANSERIFORMES & 803 & PROBOSCIDEA & 22 \\
\hline FALCONIFORMES & 2.014 & PERISSODACTYLA & 23 \\
\hline GALLIFORMES & 461 & HYRACOIDEA & 5 \\
\hline GRUIFORMES & 572 & TUBULIDENTATA & 1 \\
\hline CHARADRIIFORMES & 1.554 & ARTIODACTYLA & 369 \\
\hline COLUMBIFORMES & 503 & PHOLIDOTA & 5 \\
\hline PSITTACIFORMES & 278 & RODENTIA & 8.858 \\
\hline CUCULIFORMES & 310 & LAGOMORPHA & 280 \\
\hline STRIGIFORMES & 645 & MACROSCELIDEA & 41 \\
\hline CAPRIMULGIFORMES & 132 & & \\
\hline APODIFORMES & 1.940 & & \\
\hline COLIIFORMES & 11 & & \\
\hline TROGONIFORMES & 58 & & \\
\hline CORACIIFORMES & 712 & & \\
\hline PICIFORMES & 660 & & \\
\hline PASSERIFORMES & 11.284 & & \\
\hline NO DETERMINADO & 121 & & \\
\hline
\end{tabular}

encuentran mejor representadas las áreas pertenecientes a las antiguas colonias españolas. Los 8.996 ejemplares de España representan el $39.4 \%$ de la colección, los países latinoamericanos constituyen el $13.8 \%$, Marruecos y el Sáhara Occidental el $4.3 \%$, Filipinas el $2.2 \%$ y $2.1 \%$ los de Guinea Ecuatorial. En la Tabla 4 aparece el número de ejemplares de cada país representado.

La colección de ejemplares españoles consta de 8.996 especímenes cuyo tipo de conservación puede verse también en la Tabla 1 . Se encuentran especímenes de 65 familias y 378 especies, y están representadas todas las comunidades autónomas españolas (Tabla 5).

LA COLECCIÓN DE MAMÍFEROS.- Está formada aproximadamente por unos 27.000 ejemplares cuyo tipo de conservación aparece en la Tabla 1. El 48\% (12.984) de los ejemplares se encuentra inventariado estando el inventario totalmente informatizado. Del $52 \%$ restante está disponible un inventario preliminar a nivel de familia.

Se conservan representantes de los 20 órdenes de la clase Mammalia (Honacki et al., 1982) indicados en la Tabla 2.También están representadas 111 familias (Tabla 6), 437 géneros y 904 especies, cifras que se refieren al $48 \%$ de la colección inven- 
Tabla 3.- Número de ejemplares de las Familias de aves representadas en la colección.

\begin{tabular}{|c|c|c|c|}
\hline ACCIPITRIDAE & 1.558 & MIMIDAE & 26 \\
\hline AEGITHALIDAE & 50 & MOMOTIDAE & 26 \\
\hline ALAUDIDAE & 491 & MONARCHIDAE & 7 \\
\hline ALCEDINIDAE & 247 & MOTACILLIDAE & 333 \\
\hline ALCIDAE & 81 & MUSCICAPIDAE & 2.319 \\
\hline ANATIDAE & 786 & MUSOPHAGIDAE & 47 \\
\hline ANHIMIDAE & 13 & NECTARINIIDAE & 154 \\
\hline ANHINGIDAE & 10 & NYCTIBIIDAE & 6 \\
\hline APODIDAE & 188 & OPISTHOCOMIDAE & 5 \\
\hline APTERYGIDAE & 1 & ORIOLIDAE & 91 \\
\hline ARAMIDAE & 5 & OTIDIDAE & 91 \\
\hline ARDEIDAE & 340 & OXYRUNCIDAE & 1 \\
\hline ARTAMIDAE & 14 & PANDIONIDAE & 12 \\
\hline BOMBYCILLIDAE & 24 & PARADISAEIDAE & 46 \\
\hline BRACHYPTERACIIDAE & 3 & PARIDAE & 429 \\
\hline BUCCONIDAE & 29 & PARULIDAE & 181 \\
\hline BUCEROTIDAE & 81 & PELECANIDAE & 20 \\
\hline BURHINIDAE & 61 & PHAETHONTIDAE & 3 \\
\hline CACATUIDAE & 22 & PHALACROCORACIDAE & 63 \\
\hline CAMPEPHAGIDAE & 37 & PHASIANIDAE & 381 \\
\hline CAPITONIDAE & 94 & PHILEPITTIDAE & 6 \\
\hline CAPRIMULGIDAE & 118 & PHOENICOPTERIDAE & 23 \\
\hline CARIAMIDAE & 2 & PHOENICULIDAE & 5 \\
\hline CASUARIIDAE & 4 & PHYTOTOMIDAE & 5 \\
\hline CATHARTIDAE & 20 & PICIDAE & 392 \\
\hline CERTHIIDAE & 45 & PIPRIDAE & 102 \\
\hline CICONIIDAE & 76 & PITTIDAE & 23 \\
\hline CINCLIDAE & 49 & PLATYSTEIRIDAE & 9 \\
\hline CLIMACTERIDAE & 3 & PLOCEIDAE & 805 \\
\hline COLIIDAE & 11 & PODICIPEDIDAE & 187 \\
\hline COLUMBIDAE & 400 & POLIOPTILIDAE & 3 \\
\hline CONOPOPHAGIDAE & 1 & PROCELLARIIDAE & 69 \\
\hline CORACIIDAE & 98 & PRUNELLIDAE & 57 \\
\hline CORVIDAE & 851 & PSITTACIDAE & 224 \\
\hline COTINGIDAE & 149 & PSOPHIIDAE & 3 \\
\hline CRACIDAE & 26 & PTEROCLIDIDAE & 89 \\
\hline CRACTICIDAE & 3 & PTILONORHYNCHIDAE & 8 \\
\hline CUCULIDAE & 265 & PYCNONOTIDAE & 99 \\
\hline CHARADRIIDAE & 298 & RALLIDAE & 415 \\
\hline DENDROCOLAPTIDAE & 59 & RAMPHASTIDAE & 104 \\
\hline DICAEIDAE & 21 & RECURVIROSTRIDAE & 100 \\
\hline DICRURIDAE & 31 & REMIZIDAE & 27 \\
\hline DIOMEDEIDAE & 4 & RHABDORNITHIDAE & 2 \\
\hline DROMAIIDAE & 5 & RHEIDAE & 22 \\
\hline EMBERIZIDAE & 1.320 & RHINOCRYPTIDAE & 16 \\
\hline ESTRILDIDAE & 182 & ROSTRATULIDAE & 9 \\
\hline EURYLAIMIDAE & 12 & RYNCHOPIDAE & 5 \\
\hline EURYPYGIDAE & 1 & SAGITTARIIDAE & 2 \\
\hline FALCONIDAE & 405 & SCOLOPACIDAE & 500 \\
\hline FORMICARIIDAE & 168 & SCOPIDAE & 2 \\
\hline FREGATIDAE & 1 & SITTIDAE & 56 \\
\hline FRINGILLIDAE & 977 & SPHENISCIDAE & 9 \\
\hline FURNARIIDAE & 124 & STERCORARIIDAE & 50 \\
\hline GALBULIDAE & 22 & STRIGIDAE & 487 \\
\hline GAVIIDAE & 10 & STRUTHIONIDAE & 20 \\
\hline GLAREOLIDAE & 41 & STURNIDAE & 332 \\
\hline GRUIDAE & 32 & SULIDAE & 33 \\
\hline HAEMATOPODIDAE & 7 & SYLVIIDAE & 4 \\
\hline HELIORNITHIDAE & 2 & THINOCORIDAE & 19 \\
\hline HEMIPROCNIDAE & 4 & THRESKIORNITHIDAE & 48 \\
\hline HIRUNDINIDAE & 240 & TINAMIDAE & 36 \\
\hline HYDROBATIDAE & 17 & TODIDAE & 2 \\
\hline ICTERIDAE & 249 & TROCHILIDAE & 1.754 \\
\hline INDICATORIDAE & 1 & TROGLODYTIDAE & 107 \\
\hline IRENIDAE & 23 & TROGONIDAE & 58 \\
\hline JACANIDAE & 12 & TURDIDAE & 11 \\
\hline LANIIDAE & 339 & TURNICIDAE & 18 \\
\hline LARIDAE & 372 & TYRANNIDAE & 421 \\
\hline LORIDAE & 32 & TYTONIDAE & 155 \\
\hline MEGAPODIIDAE & 24 & UPUPIDAE & 97 \\
\hline MELIPHAGIDAE & 34 & VANGIDAE & 3 \\
\hline MEROPIDAE & 153 & VIREONIDAE & 32 \\
\hline MESITORNITHIDAE & 3 & ZOSTEROPIDAE & 23 \\
\hline
\end{tabular}

tariada y son estimativas pues aún resta por revisar taxonómicamente la colección.
Tabla 4.- Número de ejemplares de aves y mamíferos de los países representadas en la colección.

\begin{tabular}{|c|c|c|c|c|c|}
\hline & Aves & $\overline{\text { Mamiferos }}$ & & Aves & $\overline{\text { Mamíferos }}$ \\
\hline ALEMANIA & 13 & 19 & KENIA & 3 & 7 \\
\hline ANDORRA & - & 3 & LESOTHO & - & 2 \\
\hline ANGOLA & - & 1 & LIBERIA & - & 1 \\
\hline ANTILLAS & 1 & - & LIBIA & 2 & 1 \\
\hline ARGELIA & 15 & 12 & MADAGASCAR & 36 & 28 \\
\hline ARGENTINA & 50 & 417 & MALASIA & 16 & 57 \\
\hline ATLÁNTICO & - & 1 & MARRUECOS & 965 & 479 \\
\hline AUSTRALIA & 116 & 35 & MAURICIO & 1 & 1 \\
\hline AUSTRIA & 4 & 5 & MEDITERRÁNEO & - & 2 \\
\hline BAHAMAS & - & 6 & MÉXICO & 425 & 97 \\
\hline BANGLADESH & - & 1 & MICRONESIA & 4 & - \\
\hline BARBADOS & 1 & - & MOZAMBIQUE & 1 & 7 \\
\hline BIRMANIA & - & 51 & NAMIBIA & 3 & 2 \\
\hline BOLIVIA & 9 & 2 & NEPAL & 1 & - \\
\hline BOTSWANA & - & 1 & NICARAGUA & 1 & 1 \\
\hline BRASIL & 309 & 59 & NIGERIA & 8 & 1 \\
\hline CABO VERDE & - & 1 & NORUEGA & - & 7 \\
\hline CAMERÚN & 44 & 22 & NUEVA GUINEA & 40 & 13 \\
\hline CANADÁ & 4 & 13 & NUEVA ZELANDA & 10 & - \\
\hline COLOMBIA & 1.024 & 16 & PAKISTÁN & - & 2 \\
\hline COMORES & 3 & - & PALESTINA & 4 & - \\
\hline CONGO & 1 & 3 & PANAMÁ & 89 & 3 \\
\hline COSTA DE MARFIL & - & 2 & PAPUA NUEVA GUINEA & 16 & - \\
\hline COSTA RICA & 84 & 20 & PARAGUAY & 1 & 3 \\
\hline CUBA & 95 & 17 & PERÚ & 60 & 14 \\
\hline CHAD & 9 & - & POLONIA & 2 & 17 \\
\hline CHILE & 456 & 40 & PORTUGAL & 196 & 40 \\
\hline CHINA & 247 & 61 & PUERTO RICO & 13 & 3 \\
\hline CHIPRE & 1 & - & REINO UNIDO & 15 & 2 \\
\hline DINAMARCA & 26 & 1 & REP. DOMINICANA & 1 & - \\
\hline ECUADOR & 361 & 93 & RODESIA & 2 & - \\
\hline EGIPTO & 10 & 8 & RUMANIA & 31 & - \\
\hline ESPAÑA & 9.249 & 8.723 & RUSIA & 87 & 17 \\
\hline ESTADOS UNIDOS & 98 & 176 & SÁHARA OCCIDENTAL & 38 & 66 \\
\hline ETIOPÍA & 6 & 10 & SAMOA & 1 & - \\
\hline FILIPINAS & 524 & 67 & STO. TOMÉ Y PRÍNCIPE & 2 & - \\
\hline FINLANDIA & 5 & 2 & SENEGAL & 23 & - \\
\hline FRANCIA & 9 & 85 & SEYCHELLES & 1 & - \\
\hline GABON & 1 & 3 & SIERRA LEONA & 9 & - \\
\hline GAMBIA & 2 & - & SIRIA & 1 & 2 \\
\hline GHANA & 7 & 5 & SOMALIA & - & 3 \\
\hline GRAN BRETAÑA & 2 & - & SRI LANKA & 3 & 3 \\
\hline GRECIA & 5 & 4 & SUDÁFRICA & 9 & 27 \\
\hline GRENADA & 2 & 1 & SUDAN & 1 & 5 \\
\hline GUATEMALA & 227 & - & SUECIA & 31 & 5 \\
\hline GUAYANA FRANCESA & 7 & - & SUIZA & - & 16 \\
\hline GUINEA ECUATORIAL & 503 & 187 & SURINAM & - & 1 \\
\hline GUYANA & 91 & 8 & TAIWAN & 3 & 2 \\
\hline HOLANDA & 2 & - & TANZANIA & 1 & 2 \\
\hline HUNGRÍA & 9 & 1 & TRINIDAD Y TOBAGO & 20 & 2 \\
\hline INDIA & 132 & 37 & TÚNEZ & 16 & - \\
\hline INDONESIA & 151 & 59 & TURQUÍA & 3 & 13 \\
\hline IRAK & - & 22 & UGANDA & - & 4 \\
\hline IRÁN & 3 & 11 & URUGUAY & 7 & 4 \\
\hline ISLANDIA & 1 & - & VENEZUELA & 53 & 78 \\
\hline ISRAEL & 1 & - & VIETNAM & 1 & - \\
\hline ITALIA & 62 & 33 & YUGOSLAVIA & - & 1 \\
\hline JAMAICA & 17 & - & ZAIRE & 462 & 175 \\
\hline JAPÓN & 281 & 17 & ZIMBABWE & - & 2 \\
\hline JORDANIA & 2 & - & NO DETERMINADO & 7 & 2.372 \\
\hline KAZAKISTÁN & 1 & - & & & \\
\hline
\end{tabular}

En la composición geográfica se repite la misma pauta que en la colección de aves, siendo el material español el más abundante, seguido de los territorios que fueron antiguas colonias españolas. Así de España hay 7.762 ejemplares $(60 \%)$, de los países latinoamericanos 920 (7.1\%), de Marruecos y el Sáhara Occidental $545(4.2 \%)$, de Guinea Ecuatorial 187 (1.4\%) y de Filipinas sólo 67 ejemplares. En la Tabla 4 aparece el número de ejemplares de cada país representado. 
Tabla 5.- Número de ejemplares de cada una de las Comunidades Autónomas españolas representadas en las colecciones.

\begin{tabular}{lrr}
\hline & AVES & MAMÍFEROS \\
\hline ANDALUCÍA & 597 & 282 \\
ARAGÓN & 224 & 271 \\
CANARIAS & 63 & 58 \\
CANTABRIA & 98 & 511 \\
CASTILLA LA MANCHA & 2.264 & 771 \\
CASTILLA LEÓN & 1.412 & 2.770 \\
CATALUÑ & 75 & 200 \\
CEUTA & 5 & 0 \\
COMUNIDAD VALENCIANA & 393 & 141 \\
EXTREMADURA & 665 & 105 \\
GALICIA & 295 & 213 \\
ISLAS BALEARES & 86 & 696 \\
LA RIOJA & 98 & 202 \\
MADRID & 1.986 & 900 \\
MELILLA & 80 & 5 \\
NAVARRA & 54 & 176 \\
NINGUNA & 407 & 289 \\
PAÍS VASCO & 55 & 142 \\
PRINCIPADO DE ASTURIAS & 120 & 205 \\
REGIÓN DE MURCIA & 19 & 8 \\
\hline
\end{tabular}

La colección de ejemplares españoles consta de 7.736 especímenes cuyo tipo de conservación puede verse en Tabla 1. Se encuentran presentes 25 familias y 101 especies. El único territorio que no está representado es Ceuta (Tabla 5). Hemos de hacer notar que esta parte española de la colección sufrirá grandes modificaciones en cuanto a los datos expuestos, pues los especímenes de la colección general que faltan por inventariar $(52 \%)$ pertenecen mayoritariamente a material de la Península.

\section{Colecciones de Tipos}

COLECCIÓN DE AVES. - Esta colección sólo cuenta con dos ejemplares: el holotipo de un Ictérido, Icterus xantocephalus Gil, 1918, cuya validez cuestionan Sibley \& Monroe (1990) y Barreiro \& Pérez del Val (en prensa). Y con el holotipo de una subespecie de un Galliforme, Gallinula chloropus lozanoi Gil, 1918.

COLECCIÓN DE MAMÍFEROS.- Esta colección está formada por 58 ejemplares tipo, de los cuales 18 son holotipos. Se distribuyen en las siguientes familias: Arvicolidae (1), Leporidae (14), Molossidae (3), Muridae (13), Mustelidae (1), Noctilionidae (2), Phyllostomidae (5), Pteropodidae (3), Rhinolophidae (6), Sciuridae (2), Soricidae (1) y Vespertilionidae (7).
Tabla 6.- Número de ejemplares de las Familias de mamíferos representadas en la colección

\begin{tabular}{|c|c|c|c|}
\hline ABROCOMIDAE & 3 & MACROPODIDAE & 12 \\
\hline AGOUTIDAE & 1 & MACROSCELIDIDAE & 33 \\
\hline ANOMALURIDAE & 7 & MANIDAE & 5 \\
\hline APLODONTIDAE & 1 & MEGADERMATIDAE & 4 \\
\hline ARVICOLIDAE & 2.599 & MICROBIOTHERIIDAE & 1 \\
\hline BATHYERGIDAE & 2 & MOLOSSIDAE & 72 \\
\hline BOVIDAE & 221 & MONODONTIDAE & 5 \\
\hline BRADYPODIDAE & 4 & MORMOOPIDAE & 2 \\
\hline CAENOLESTIDAE & 5 & MURIDAE & 3.738 \\
\hline CALLITHRICIDAE & 31 & MUSTELIDAE & 480 \\
\hline CAMELIDAE & 4 & MYOCASTORIDAE & 10 \\
\hline CANIDAE & 137 & MYRMECOBIIDAE & 1 \\
\hline CAPROMYIDAE & 2 & MYRMECOPHAGIDAE & 9 \\
\hline CASTORIDAE & 1 & NOCTILIONIDAE & 11 \\
\hline CAVIIDAE & 11 & NYCTERIDAE & 5 \\
\hline CEBIDAE & 62 & OCTODONTIDAE & 12 \\
\hline CERCOPITHECIDAE & 95 & ODOBENIDAE & 8 \\
\hline CERVIDAE & 62 & ORNITHORHYNCHIDAE & 2 \\
\hline CRICETIDAE & 410 & ORYCTEROPODIDAE & 1 \\
\hline CTENOMYIDAE & 302 & OTARIIDAE & 7 \\
\hline CYNOCEPHALIDAE & 8 & PETAURIDAE & 3 \\
\hline CHEIROGALEIDAE & 3 & PHALANGERIDAE & 10 \\
\hline CHINCHILLIDAE & 7 & PHASCOLARCTIDAE & 3 \\
\hline CHOLOEPIDAE & 7 & PHOCIDAE & 8 \\
\hline CHRYSOCHLORIDAE & 2 & PHOCOENIDAE & 2 \\
\hline DASYPODIDAE & 22 & PHYLLOSTOMIDAE & 84 \\
\hline DASYPROCTIDAE & 5 & PHYSETERIDAE & 1 \\
\hline DASYURIDAE & 5 & PONGIDAE & 28 \\
\hline DAUBENTONIIDAE & 2 & PROCAVIIDAE & 5 \\
\hline DELPHINIDAE & 11 & PROCYONIDAE & 19 \\
\hline DIDELPHIDAE & 25 & PTEROPODIDAE & 62 \\
\hline DIPODIDAE & 35 & RHINOCEROTIDAE & 12 \\
\hline DUGONGIDAE & 1 & RHINOLOPHIDAE & 388 \\
\hline ELEPHANTIDAE & 22 & RHIZOMYIDAE & 5 \\
\hline EMBALLONURIDAE & 22 & SCIURIDAE & 574 \\
\hline EQUIDAE & 9 & SORICIDAE & 322 \\
\hline ERETHIZONTIDAE & 5 & SUIDAE & 28 \\
\hline ERINACEIDAE & 228 & TACHYGLOSSIDAE & 3 \\
\hline FELIDAE & 163 & TALPIDAE & 555 \\
\hline GALAGIDAE & 3 & TAPIRIDAE & 2 \\
\hline GEOMYIDAE & 10 & TARSIIDAE & 1 \\
\hline GIRAFFIDAE & 9 & TAYASSUIDAE & 3 \\
\hline GLIRIDAE & 1.006 & TENRECIDAE & 4 \\
\hline HERPESTIDAE & 19 & THYLACINIDAE & 1 \\
\hline HETEROMYIDAE & 43 & THYLACOMYIDAE & 1 \\
\hline HIDROCHAERIDAE & 2 & THYROPTERIDAE & 3 \\
\hline HIPPOPOTAMIDAE & 3 & TRAGULIDAE & 10 \\
\hline HOMINIDAE & 4 & TRICHECHIDAE & 2 \\
\hline HYAENIDAE & 4 & TUPAIIDAE & 16 \\
\hline HYDROCHAERIDAE & 1 & URSIDAE & 26 \\
\hline HYLOBATIDAE & 3 & VESPERTILIONIDAE & 933 \\
\hline HYSTRICIDAE & 13 & VIVERRIDAE & 300 \\
\hline INDRIIDAE & 5 & VOMBATIDAE & 1 \\
\hline LEMURIDAE & 13 & ZAPODIDAE & 4 \\
\hline LEPORIDAE & 261 & ZIPHIIDAE & 1 \\
\hline LORISIDAE & 2 & NO DETERMINADO & 203 \\
\hline
\end{tabular}

\section{Publicaciones sobre la Colección}

ColeCCión DE AVES. - De esta colección no existe ningún catálogo publicado, el único que se conserva es el manuscrito realizado por M.P. Graells en 1846 que se guarda en el archivo histórico del Museo. Actualmente estamos en proceso de revisión taxonómica y la publicación del Volumen I del catálogo general referido a las pieles de estudio de las aves no Passeriformes que se publicará previsiblemente el año 1988 (Barreiro et al., en prensa). Sobre algunos ejemplares existen publicaciones puntuales (Gil Lletget, 1918a, 1918b, 1941, 1943), 
pero sólo A. Gil Lletget en 1935 publica un catálogo de una pequeña parte, los Ploceidos del Congo Belga.

COLECCIÓN DE MAMífERos.- Además del catálogo manuscrito realizado por M.P. Graells en 1846, A. Cabrera publicó el Catálogo de Tipos de la colección en 1912, y de ese mismo año data el Catálogo Metódico de los fondos, obra asimismo de este autor. A. Cabrera publicó también el catálogo de la colección de los monos americanos en 1900, y los mamíferos del viaje al Pacífico en 1917. E. Morales Agacino (1935a, 1935b), publicó la lista de los mamíferos capturados en Luluabourg (ex Congo Belga) y la de los capturados en Ifni.

Más modernamente se han publicado el Catálogo de los Murciélagos (Ibáñez \& Fernández, 1989) y una lista de mamíferos con valor histórico (Delibes \& García-Valdecasas, 1987).

\section{Facilidades}

El sistema de gestión de las colecciones de aves y mamíferos del Museo es similar para ambas. La descripción del funcionamiento de este sistema se puede encontrar en Barreiro et al. (1994). El programa Access 2.0 de Microsoft es el que estamos empleando actualmente en la informatización de las bases de datos de las colecciones.

En los almacenes las colecciones se encuentran ordenadas por orden sistemático de ordenes y familias, los géneros y las especies se guardan en orden alfabético. Por cuestiones puramente prácticas se emplea un libro de referencia en la ordenación y nomenclatura de los especímenes en cada colección. Para las aves utilizamos Howard y Moore (1991) y en mamíferos Honacki et al. (1982).

Para solicitar una consulta o un préstamo a las colecciones, según se indica en la normativa del Centro (Sanchiz, 1994), deben ponerse en contacto con la conservadora con suficiente antelación. Como es habitual en estas colecciones, los ejemplares tipos no son objeto de préstamo, teniendo que ser consultados en las instalaciones del Museo.

\section{AGRADECIMIENTOS}

Deseo agradecer la ayuda prestada al Dr. J. Pérez del Val y a la Dra. Ana I. Camacho en la redacción de este trabajo; a Miguel Villena y Javier Campos por su paciencia al explicarme el manejo de los programas informáticos; $\mathrm{y}$ a todas las personas que han contribuido a lo largo de los años en la ardua tarea de la realización del inventario.

\section{Referencias}

Barreiro, A.J., 1992. El Museo Nacional de Ciencias Naturales (1771-1935). Ediciones Doce Calles. Aranjuez. 509 pp.

Barreiro, J., GonzÁlez FernÁndez, J.E. \& ReyFRAILE, I., 1994. Las colecciones de vertebrados: uso y gestión. En: B. Sanchiz (ed.). Manual de catalogación y gestión de las colecciones científicas de Historia Natural. Manuales Técnicos de Museología. Vol. 5. Museo Nacional de Ciencias Naturales. CSIC. Madrid: 21-80.

Barreiro, J. \& Pérez del Val, J., (en prensa). Catálogo de las Colecciones de Aves del Museo Nacional de Ciencias Naturales de Madrid. Vol. I. Aves No Passeriformes (Pieles de Estudio). Manuales Técnicos de Museología. Museo Nacional de Ciencias Naturales. CSIC. Madrid.

Barreiro, J. \& Pérez del VAl, J., (en prensa). Icterus xantolaemus Gil, 1918, a non valid species.

CABrera, A., 1900. Estudios sobre una colección de monos americanos. An. Soc. Esp. Hist. Nat., 29: 6493.

CAbrera, A., 1912. El concepto de tipo en Zoología y los tipos de mamíferos del Museo de ciencias Naturales. Trab. Mus. Cienc. Nat., 3: 3-32.

Cabrera, A., 1912. Catálogo Metódico de las colecciones de mamíferos del Museo Nacional de Ciencias Naturales de Madrid. Trab. Mus. Cienc. Nat., 11: 1147.

Cabrera, A., 1917. Mamíferos del Viaje al Pacífico. Trab. Mus. Nac. Cienc. Nat., Ser. Zool., 31: 3-62.

Delibes, J. \& García-Valdecasas, A., 1987. Museo Nacional de Ciencias Naturales, Spain: Specimens of Historical Significance. En: Genoways, H; Jones, C \& Rossolimo, O. (eds.). Mammal Collection Management. Texas Tech Univ. Pess. Lubbock, Texas: $179-184$

Gil Lletget, A., 1918a. La Gallinula chloropus de Filipinas. Bol. R.. Soc. Esp. Hist. Nat. 18: 75-77.

Gil Lletget, A., 1918b. Dos Passeres nuevos de la colección de la Expedición al Pacífico. Bol. R. Soc. Esp. Hist. Nat., 18: 340-341.

Gil Lletget, A., 1935. Aves del Congo Belga: I. Fam. Ploceidae. Bol. Soc. Esp. Hist. Nat., 35: 69-184.

Gil Lletget, A., 1941. Sobre un Momótido nuevo del Ecuador. Bol. Soc. Esp. Hist. Nat., 39: 337-338.

Gil Lletget, A., 1943. Muscicápidos de Luluabourg (Congo Belga) existentes en el Museo de Madrid. Bol. Soc. Esp. Hist. Nat., 41: 179-190.

Graells, M.P., 1846. Catálogo de las colecciones de Anatomía Comparada. Mamíferos, Aves, Reptiles y Peces del Museo Nacional de Ciencias Naturales de Madrid. Manuscrito inédito. Archivo Histórico del Museo Nacional de Ciencias Naturales. 
Honacki, J.H., KinMan, K.E. \& KoEPPL, J.W., (Eds.) 1982. Mammal species of the world. A taxonomic and geographic reference. Allen Press \& The Association of Systematics Collections. Lawrence, Kansas, USA. 694 pp.

Howard, R. \& Moore, A. 1991. A Complete Checklist of the Birds of the World. Academic Press. Londres. $622 \mathrm{pp}$.

IbÁÑEZ, C. \& FERnÁNDEZ, R., 1989. Catálogo de Murciélagos de las Colecciones del Museo Nacional de Ciencias Naturales. Monografías del Museo Nacional de Ciencias Naturales. CSIC. Madrid. 54 pp.

Morales Agacino, E., 1935a. Sobre algunos insectívoros de Saint Joseph de Luluabourg (Congo Belga). Bol. Soc. Esp. Hist. Nat., 35:17-23.

Morales Agacino, E., 1935b. Mamíferos de Ifni. Bol. Soc. Esp. Hist. Nat., 35: 381-393.
Peters, J.L., 1931-1987. Checklist of the birds of the world. Vols. I-XVI. Harvard Univ. Press. Cambridge. USA.

SAnchiz, B., 1994. Anexo: Normativa general de las colecciones científicas del Museo Nacional de Ciencias Naturales. En: B. Sanchiz (ed.). Manual de catalogación y gestión de las colecciones cientificas de Historia Natural. Manuales Técnicos de Museología. Vol. 5. Museo Nacional de Ciencias Naturales. CSIC. Madrid: 225-229. 